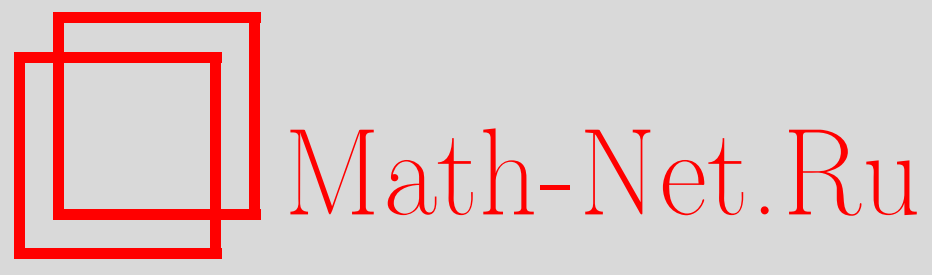

Е. В. Панфилова, Новые книги, Теория вероятн. и ее примен., 2005, том 50, выпуск 2, 414

DOI: https://doi.org/10.4213/tvp122

Использование Общероссийского математического портала Math-Net.Ru подразумевает, что вы прочитали и согласны с пользовательским соглашением

http://www . mathnet.ru/rus/agreement

Параметры загрузки:

IP : 3.89.185.249

26 апреля 2023 г., 14:29:30

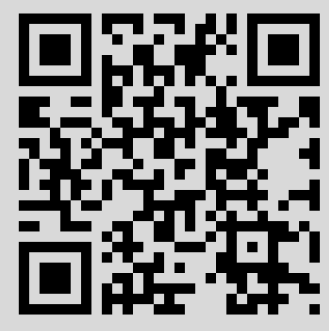




\section{НОВЫЕ КНИГИ}

Activity-based statistics: Instructor resources. Ed by $\mathrm{R}$. Scheaffer, V. Gnanadesikan, A. Watkins, J. Witmer. 2nd. ed., Springer-Verlag, Berlin, 2004, 300 p. Activity-based statistics: Student guide. Ed by R. Scheaffer, V. Gnanadesikan, A. Watkins, J. Witmer. 2nd. ed., Springer-Verlag, Berlin, 2004, 300 p.

A s m u s s e n S. Applied probability and queues. 2nd. ed, Springer, New York, 2003, xii, 438 p.

B e $\mathbf{n ~ t ~ h ~ F . ~ E . ~ O p t i o n ~ t h e o r y ~ w i t h ~ s t o c h a s t i c ~ a n a l y s i s . ~ A n ~ i n t r o d u c t i o n s ~ t o ~}$ mathematical finance. Springer, Berlin, 2004, x, 162 p.

C a r m o n a R. A. Statistical analysis of financial data in S-PLUS. Springer texts in statistics. Springer, Berlin, 2004, xvi, $451 \mathrm{p}$.

C h u n g K. L., A i t $\mathbf{S}$ a h $\mathbf{l}$ i a F. Elementary probability theory. With stochastic processes and an introduction to mathematical finance. 4th ed. Springer, Berlin, 2003, xiii, 402 p.

CreditRisk + in the banking industry. Ed. by M. Gundlach, F. Lehrbass. Springer, Berlin, 2004, 369 p.

D a t t a G. S., M u k e r j e e R. Probability matching priors: Higher order asymptotics. Lecture notes in statistics 178. Springer, New York, 2004, x, $127 \mathrm{p}$.

$\mathbf{F} \mathbf{r}$ a $\mathbf{n}$ k e J., $\quad \mathbf{H}$ ä $\mathbf{r} \mathbf{d} \mathbf{l}$ e W., $\mathbf{H}$ a $\mathbf{f} \mathbf{n}$ e $\mathbf{r}$ C. M. Introduction to the statistics of financial markets. Springer, Berlin, 2004, xxi, $428 \mathrm{p}$.

G i k h m a n I. I., S $\mathbf{k}$ or o k hod A. V. The theory of stochastic processes I; II; Reprint of the 1st ed. 1974; 1975. Springer, Berlin, 2004, x, 578 p.; x, $441 \mathrm{p}$.

G o h $\mathbf{m} \mathbf{R}$. Noncommutative stationary processes. Lecture notes in mathematics 1839. Springer, Berlin, 2004, vii, 170 p.

I t ô K. Stochastic processes. Lectures given at Aarhus University. Ed. and with a foreword O. E. Barndorff-Nielsen, K. Sato. Springer, Berlin, 2004, xii, 234 p.

$\mathbf{J}$ a c o d J., P r o t t er Ph. Probability essentials. 2nd revised ed. Springer, Berlin, 2004, x, $254 \mathrm{p}$.

K e s t e n H. Probability on discrete structures. Encyclopaedia of mathematical sciences 110. Springer, Berlin, 2004, ix, $351 \mathrm{p}$.

$\mathrm{M}$ a x w e $11 \mathrm{~N}$. Data matters. Conceptual statistics for a random world. Key Colledge Publishing, Emeryville, CA, 2004, xiv, 625 p.

$\mathbf{P} \mathbf{r} \circ \mathbf{t} \mathbf{e} \mathbf{r} \mathbf{P h}$. Stochastic integration and differential equations. 2nd ed. Applications of mathematics 21. Springer, Berlin, 2004, xiii, $415 \mathrm{p}$.

$R \mathbf{u} p \mathbf{p}$ e $\mathbf{r} \mathbf{t}$ D. Statistics and finance: An introduction. Springer texts in statistics. Springer, Berlin, 2004, xii, 482 p.

S h $\mathbf{r}$ e v e S. E. Stochastic calculus models for finance I. The binomial asset pricing model. Springer, Berlin, 2004, $216 \mathrm{p}$.

$\mathbf{S} \mathbf{h} \mathbf{r}$ e $v$ e S. E. Stochastic calculus models for finance II. Continuous-time models. Springer, Berlin, 2004, 550 p.

S i l v e s t r o v D. S. Limit theorems for randomly stopped stochastic processes. Probability and its Applications. Springer, London, 2004, xiv, 398 p.

$\mathbf{S ~ k ~ o ~ r ~ o ~ k ~ h ~ o d ~ A . ~ V . ~ B a s i c ~ p r i n c i p l e s ~ a n d ~ a p p l i c a t i o n s ~ o f ~ p r o b a b i l i t y ~ t h e o r y . ~}$ Springer, New York, 2004, 260 p.

d e S o u z a P. N., S i l v a J.-N. Berkeley problems in mathematics. 3rd ed. Springer, New York, 2004, xiv, 591 p.

W a s s e $\mathbf{r} \mathbf{m}$ a $\mathbf{n}$ L. All of statistics. A concise course in statistical inference. Springer, New York, 2004, xix, 442 p. 